\title{
- BOOK REVIEWS -
}

\section{- An EMDR Therapy Primer, Second Edition: From Practicum to Practice}

\author{
Barbara J. Hensley. New York, NY: Springer \\ Publishing Company, 2015, 481 pp., $\$ 65.00$ \\ (paperback); $\$ 64.99$ (kindle or nook)
}

I consider Barbara Hensley's EMDR Therapy Primer, Second Edition: From Practicum to Practice to be a foundational text, complementing Francine Shapiro's 2001 text and eye movement desensitization and reprocessing (EMDR) therapy basic training course. This extensively revised and expanded edition represents the evolution of EMDR therapy theory, practice, and research. Written to assist newly trained EMDR clinicians in their understanding, skills, and integration of EMDR, the Primer also serves as a refresher for clinicians who have not kept abreast of the refinements in EMDR therapy or want to review the basics.

This comprehensive and practical guide to EMDR therapy fundamentals is readable and well written. Chapter 1 provides an overview of the adaptive information processing (AIP) model, potential mechanisms of action, and methods, including the eight phases and three pronged approach. I appreciate the book's updated summary of research, thorough discussion of bilateral stimulation, and coverage of key concepts.

Because it is NOT a manualized therapy, I think it is essential that EMDR clinicians understand the why (theoretical model) and when (treatment plan) as well as the how (methods) of EMDR therapy. Chapter 2 grounds the eight phases within the AIP model, describing the goals and procedures of each phase. Chapter 3 explains effective target selection, the how and why of Phase 3 assessment of a target memory, and protocols for recent and single-incident traumas.

I have noticed a common misconception by clients and therapists that clearing the past alone should resolve the presenting issue. Chapter 4 explores the three pronged approach-working with the past, present, and future. To assist in case formulation and treatment planning, methods are described to identify experiences that contribute to current symptoms. Clear guidance and rich clinical examples are given for targeting past memories and present triggers and developing positive templates for the future.

In my personal copy of this text, the tables in Chapter 5 are becoming well-worn pages. Dr. Hensley explains what is occurring when active reprocessing is not progressing and offers tables listing various AIP informed interventions, including cognitive interweaves. Deany Laliotis and Deborah Korn present eight categories of interweaves: experiential, modulation, defense, developmental repair, relational, information, integration, and action interweaves. More than 100 brief statements, questions, or suggestions are listed as examples.

Although Chapter 2 recommends that therapists learn about and screen for dissociation, I think that clinicians would have benefited from descriptions of how dissociation can impact each of the eight phases. For example, Chapter 5 could have included guidance on how to recognize and address dissociation when it contributes to intense emotional processing, overaccessing, underaccessing, and blocked processing as well as indicators that a clinician should consider the presence of a dissociative disorder.

This edition's revisions and additions are extensive. In the session transcripts in Chapter 6, additional cases demonstrate the use of eye movement desensitization (EMD) and cognitive interweaves. Updated instructions-from $\mathrm{calm} /$ safe place to future templates - provide clearer guidance. The tables of errors and obstacles to therapeutic success called "derailment possibilities" and new instructive tables, illustrations, scripts, and clinical case examples elucidate essential teaching points and enrich every chapter.

This step-by-step guide complements and expands on the EMDR therapy basic training course, fills in any gaps or areas of confusion, and provides guidance for clinicians wanting to cultivate their understanding and skills. Whether trained recently or long ago, readers will find this back-to-basics text a valuable refresher and accessible reference to increase confidence and enhance their fidelity to the AIP model and evidence-based methods of EMDR therapy.

REVIEWED BY KATY MURRAY 


\section{- EMDR Therapy for Clinicians Self-Care: Models, Scripted Protocols, and Summary Sheets for Mental Health Interventions}

Marilyn Luber, New York, NY: Springer Publishing, 2015, 83 pp., \$30.00 (paperback)

This comprehensive workbook presents four brief yet dense chapters that succinctly outline four different approaches used by eye movement desensitization and reprocessing (EMDR)-focused community disaster responders intended to minimize vicarious trauma. Each chapter includes a summarized checklist, which equips clinicians with a self-assessment tool intended to identify signs of vicarious trauma. The primary purpose of the book is to provide an accessible self-care guide for mental health care clinicians involved in community disaster relief.

In Chapter 1, Neil Daniels, director of the Post Traumatic Stress Disorder (PTSD) Clinical Team at the Philadelphia VA Medical Center argues that community crisis response teams must first prepare themselves by way of having a clear self-care plan before they can effectively treat trauma victims. Thereby, Daniels offers a protocol he designed for therapists' self-care following clinical sessions ending with unresolved negative affect.

The second chapter, authored by Karen Alter-Reid, describes the "Therapy for Therapists" protocol, which was developed by a team of Trauma Recovery Network responders. Before local practitioners are trained in EMDR therapy so that they can respond to community disasters the trainers are provided with EMDR therapy. It is assumed that these mental health care providers may have also been traumatized by the tragic community events to which they will be responding. For example, providers may also be traumatized by community tragic events to which they are responding. Alter-Reid emphatically asserts that professional responders needs are as important as those of disaster victims.

Derek Farrell, president of EMDR-HAP (Europe Humanitarian Assistance Programs), in Chapter 3, offers his personal reflections on the inevitability of vicarious trauma among responders, and thus the need for onsite EMDR clinical training and supervision. He discloses when he attempted to integrate back into his personal professional life, after responding to the devastating 1999 Turkish earthquake, he became painfully aware of destabilizing symptoms resulting from vicarious trauma. He writes, "Probably the most important healing aspect, initially, was in experiencing a profound reality check that what I was undergoing was actually understandable and was already known about in academic literature." (p. 25) He highlights pervasive symptoms and attributes of vicarious trauma and clearly describes parallels with PTSD.

Farrell's protocol delineates concise action points for consideration by clinicians and other professionals involved in on-the-ground humanitarian response work. His chapter concludes with an EMDR group exercise and several worksheets intended to enhance clinicians' otherwise compromised well-being and provide support.

Finally, Chapter 4, "Worst Case Scenarios in Recent Trauma Response," takes a practical proactive approach, recommending preparation by natural disaster responders prior to their deployment. For example, included is a comprehensive list of practical supplies and proactive actions necessary for emergency trauma-related response work. Some suggestions may be viewed as less realistic than idealistic. For instance, Jarero and Uribe suggest that responders "settle professional and domestic issues" (p. 46) prior to traveling to a disaster site to assure mental stability and focus on the immediate concerns involving victims and care providers.

Overall, editor Marilyn Luber's text, EMDR Therapy for Clinical Self-Care, is a standard EMDR and adaptive information processing (AIP) oriented resource for mental health interventions intended to advance support and well-being for clinicians working in disaster response and their EMDRtherapist-trainees. Some of the therapist self-care material may have a more general application. Likewise, her book may speak to a wider audience of EMDR therapy trainees, certified trainers, and experienced EMDR therapy clinicians. Particularly for beginners of EMDR therapy, this text is a valuable resource given its contents, models, scripted protocols, and summary sheets. On the other hand, experienced EMDR therapists may appreciate its emphasis on self-care. Overall, EMDR therapists may appreciate Editor Luber's book as an informative resource in EMDR therapists' library.

REVIEWED BY PAMELA ASHKENAZY

\section{- EMDR With First Responders: Models, Scripted Protocols, and Summary Sheets for Mental Health Interventions}

Marilyn Luber. New York, NY: Springer Publishing Company, 2015, 189 pp., \$29.99 (Kindle), $\$ 30.00$ (paperback)

If you would like a road map on how to integrate eye movement desensitization and reprocessing (EMDR) into your community to support those who respond so the rest of us can go about our lives, then look no further. In this user-friendly hybrid between text and manual, Marilyn Luber continues her tradition of gathering reliable technical information and practical worksheets and embedding them in adaptive information processing terms that any well-trained EMDR clinician can understand.

The book is arranged in two main parts. Part one focuses on the use of EMDR early on following a traumatic event with first responders. The types of first responders include firefighters, police officers, and emergency medical technicians, while also some professionals who may not come readily to mind. These include medical professionals 
and protective services workers. If you are a clinician practicing EMDR in the community where you live, it will be hard to walk away from this book without starting your own outreach project to your favorite group of neighbors serving your community. If you have already begun reaching out, you will sigh with relief as you learn from those who have gone before you to bring EMDR home. Part two takes a look at early interventions with EMDR for special situations including mining accidents and scenarios where it is necessary to limit client disclosure of the events. Although this section is limited in variety, EMDR clinicians serving their own unique community of workers will gain insight from the scaffolding that emerges as the authors reveal their innovative use of early EMDR interventions for those working "off the beaten path."

The authors are well chosen and all convey a sense of telescopic precision at orienting clinicians to micro and macro issues. At one glance, common negative cognitions of police officers dealing with line of duty deaths are covered, and at the next, global features related to law enforcement culture like the administrative concerns of a law enforcement agency that will influence the treatment frame. Among the contributors are Robbie Adler-Tapia, Marilyn Luber, Roger Solomon, Mark Russell, Tammera Cooke, Susan Rogers, and David Blore. The authors make the assumption that adapting the standard eight-phase EMDR protocol lends itself to addressing the needs of first responders dealing with acute stress disorder (ASD) while being transparent to acknowledge the evidence that does exist and highlight the overwhelming evidence of the standard protocol's efficacy in treating posttraumatic stress disorder (PTSD). Nonetheless, the authors reveal that the ubiquitous use of these interventions around the world is compelling. This coupled with their honest portrayal of the scientific evidence places this book solidly in the canon of works destined to illuminate the debate over ASD, PTSD, and psychotherapy.

The chapters vary with quantity and quality of case examples. This may take away from the story telling excitement that some psychotherapy books have. If you are a reader looking to get some work done, however, and not just be entertained, I recommend you place this one squarely in your EMDR gig bag and get out there!

\section{REVIEWED BY TONY COTRACCIA}

\section{- EMDR Therapy for Schizophrenia and Other Psychoses}

Paul William Miller. New York, NY: Springer Publishing Company, 2016, 261 pp., £42.99 (paperback), £40.99 (kindle)

As a psychiatrist investigating outcomes of eye movement desensitization and reprocessing (EMDR) provision for patients with psychosis, I found Paul Miller's book, EMDR
Therapy for Schizophrenia and Other Psychoses exciting, and it went far beyond my expectations. The sheer extent of the evidence and consideration into this topic from areas such as neurobiology, mindfulness practice, clinical and historical psychiatry, as well as the psychodynamic and cognitive behavioral psychotherapeutic modalities has been awe-inspiring. For a book proposing a method that in some circles is still considered uninformed and risky, it more than adequately informs the audience as to the evolving clinical, experiential, neurobiological, and research evidence to inform the safe and effective treatment of patients who are often left with little hope. Although written for clinicians and researchers who are experienced in both EMDR therapy and the treatment of psychotic illness, the author's warm narrative style, with frequent examples and the eclectic use of quotes, resources, and references, helps make it accessible to a wider audience.

The foreword, preface, and introduction pique your interest and signpost you through the book. An exploration through psychiatry's history ultimately assures you of the valid link between psychosis and dissociative experiences (an area which has also grown significantly with the integration of ego state therapy and EMDR). Next, a psychiatrically informed assessment process for a patient with psychosis integrates well and reinforces the adaptive information processing (AIP) model. The chapter on case conceptualization provides useful, informed analogies as to the rationale and process of targeting the dysfunctional memory networks which lie behind the presenting symptoms. These are described as the fruit of the hidden dysfunction which was initially adaptively shrouded behind the curtain of psychosis. The mythological meme of Dorothy being led behind the wizard's curtain in The Wizard of $\mathrm{Oz}$ is one of the gems of analogy that help you get alongside patients without dismissing their symptoms or difficulties. A concern of mine on researching this subject myself has been the lack of use of the "affect bridge" in the development of targets, but this author uses it as a core skill to identify targets.

At this point, I was fearful of a simple rehashing of the EMDR protocol. However, the Gestalt of psychotic presentation and experience is introduced successfully and appears to helpfully widen the application of EMDR processing to thematic material rather than clear targets. The author's proposed model for case conceptualization and treatment provision is clearly derived from experience working with this client group and provides approaches that help reveal or access what is behind the "curtain" of psychosis. Treatment is explained intuitively while remaining held within the context of psychodynamic and relational psychotherapeutic work. This adds a warm and validating experience for an EMDR therapist reading the book because it provides such a cogent rationale for embedding EMDR in a wider therapeutic milieu.

In his next edition, I hope that the author can extend the case formulation strategies even further, in consideration to flash-forward, and provide some discussion on the 
management of psychotic experiences which cannot be conversed with. I felt the book could have benefited from even more case examples and an in-depth description of the full course of the EMDR therapy with a patient.

In my view, this book is beautifully written in small chunks, with helpful reiteration and repetition throughout the book to really let the message and evidence sink in. And the potentially dry, academic subject is warmed up with quotes as widely sourced from Buddhist masters to J. K. Rowling. This book is an inspiration for EMDR therapists and professionals who work with clients with psychosis because it places us in an opportune moment in history to improve the lives of these patients. It provides me such hope that over my career, we can establish this way of working and travel alongside patients through their personal journey to heal and reintegrate dysfunction.

\section{REVIEWED BY SIMON MARLOW}

\section{- EMDR Journal: A Companion for Healing, Courage, and Clarity}

Colleen Patrice, Spiritual Psychology Trauma Survivor, Colleen Patrice, 2010, 229 pp., \$24.95 (softcover)

Colleen Patrice, MA, has developed a useful concept in a supplemental resource for eye movement desensitization and reprocessing (EMDR) therapy with the idea of an introspective companion journal for the client. Although the author had a good idea in creating EMDR Journal: $A$ Companion for Healing, Courage, and Clarity to supplement EMDR therapy, more attention to detail in editing and content would have increased the overall value of this book.

Following a brief introduction about the author and her experiences with trauma and EMDR, the book begins with a highly condensed overview of EMDR. In just 10 pages, Patrice outlines 15 section titles such as "What is EMDR?" "How Does EMDR Work?" and "After EMDR comes RDI." In these sections, Patrice writes about concepts addressed in therapy. A strength of the overview is that it describes what to expect directly following an EMDR session. Especially informative is Patrice's section entitled "A Few Notes/Suggestions," in which she offers tips for understanding trauma in clear, nonjargon language.

The remainder of the book is broken into categories such as "Preparing for your First EMDR Session" and "Directly after Your First EMDR Session." These sections offer questions such as "Do you notice any negative beliefs you might be holding about yourself?" "Do you remember any dreams?" and "Please acknowledge yourself for three things you did well today." This format is repeated throughout the book with blank lines available for journaling.

A downside of this book is that Patrice does not reference the adaptive information processing (AIP) model or Francine Shapiro's standard protocol. However, she does direct the reader to www.emdria.org for information about EMDR. Patrice devotes the four final sections to resource development installation (RDI), defining RDI as a stage to be completed following EMDR.

Fundamentally, the representation of RDI is inaccurate based on Francine Shapiro's standard protocol (2001, p. 472) and Andrew Leeds description of RDI as defined in his 2009 book (p. 410). Because RDI is neither a phase of EMDR nor is it a criterion of post-EMDR planning, it appears that Patrice is describing future template instead of RDI. Achieving clarity to this point in future editions would be advantageous to this workbook.

This idea of a companion journal to supplement EMDR is an excellent one, and Patrice has offered a casual, easy-to-read format. Nonetheless, improved editing and attention to content would have improved the book's value as an informative and educational resource for both the clinician and the client.

REVIEWED BY STACEY STEVENS

\section{References}

Leeds, A. (2009). A guide to the standard EMDR protocols for clinicians, supervisors, and consultants. New York, NY: Springer Publishing.

Shapiro, F. (2001). Eye movement desensitization and reprocessing: Basic principles, protocols, and procedures (2nd ed.). New York, NY: Guilford Press. 